\title{
Statistical Analyses of Turbulent Particle and Momentum Fluxes in a Cylindrical Magnetized Plasma*)
}

\author{
Makoto SASAKI ${ }^{1,2)}$, Naohiro KASUYA ${ }^{1,2)}$, Masatoshi YAGI ${ }^{2,3)}$, Kimitaka ITOH ${ }^{2,4)}$, \\ Yoshihiko NAGASHIMA ${ }^{1,2)}$, Shigeru INAGAKI ${ }^{1,2)}$ and Sanae-I. ITOH ${ }^{1,2)}$ \\ ${ }^{1)}$ Research Institute for Applied Mechanics, Kyushu University, Kasuga 816-8580, Japan \\ ${ }^{2)}$ Itoh Research Center for Plasma Turbulence, Kyushu University, Kasuga 816-8580, Japan \\ 3) Japan Atomic Energy Agency, Rokkasyo-Mura, Aomori 039-3212, Japan \\ 4) National Institute for Fusion Science, Toki, Gifu 509-5292, Japan
}

(Received 7 December 2012 / Accepted 21 June 2013)

\begin{abstract}
A nonlinear simulation of resistive drift wave turbulence in a cylindrical plasma is carried out. Long time evolution of turbulence with formation of a zonal flow is obtained for more than 1000 times of the typical drift wave period, which is sufficient for statistical analyses. Dynamical particle and momentum balance for the formation of the mean reveals that the radial turbulent fluxes are dominant contributors for the evolution of fluctuations. The particle flux is found to precede the momentum flux for 0.4 times of the typical drift wave period with large temporal variance. The analyses of the time series data of 3-D fields give the understanding of the dynamical structural formation mechanism.
\end{abstract}

(C) 2013 The Japan Society of Plasma Science and Nuclear Fusion Research

Keywords: drift wave turbulence, cylindrical magnetized plasma, zonal flow, particle flux, momentum flux, probability density function

DOI: $10.1585 /$ pfr.8.2401113

\section{Introduction}

Large efforts in the magnetic confinement fusion theory have been devoted to evaluating turbulent particle and momentum transport arising from various kinds of microinstabilities [1]. One of the conventional approaches to this problem is taken by calculating the mean contribution to obtain the transport coefficient. However, it is clear that plasma turbulence involves bursty events, which is intermittent in time [2]. Probability density function (PDF) is one of the methods to quantify such kind of contribution. Theories and numerical simulations on PDFs of turbulent fluctuations is progressing, and cause of deviation from Gaussian PDF is studied [3,4]. PDFs are also used in experimental researches [5]. Recently, simultaneous measurements of particle and momentum fluxes in cylindrical plasmas shows correlation between particle and momentum fluxes [6]. Turbulent structures are formed with the balance of particle and momentum fluxes, so the contribution from intermittent events must be clarified. Turbulence diagnostic simulator (TDS) is an assembly of simulation codes to clarify the formation mechanism of turbulent structures by numerical diagnostics [7]. Structural formation mechanisms in cylindrical plasmas have been studied using the TDS [8]. The simulation model in this study is based on the extension of Hasegawa-Wakatani equations [9]. There are lots of studies on turbulence by Hasegawa-

author'se-mail: sasaki@riam.kyushu-u.ac.jp

*) This article is based on the presentation at the 22nd International Toki Conference (ITC22).
Wakatani equations. The generation of zonal flows (ZFs) is predicted [10], and importance of three-dimensional properties of turbulence has been pointed out [11-13]. The individual statistical properties of fluctuations of physical variables and their fluxes have been investigated [4].

In this study, we focus on the statistical properties of the link between fluctuations of physical variables and their fluxes, and interference between fluxes. We perform a numerical simulation of drift wave turbulence in a cylindrical plasma using the numerical linear device (NLD) [14], which is the part of the TDS. Nonlinear saturation with formation of a zonal flow is obtained in the simulation, and statistical characteristics of particle and momentum fluxes are investigated. The global simulation gives the comparison of the characteristics at different radii, and the dominant contribution to the variation of the mean profile is identified. The correlation between the particle and momentum fluxes is also evaluated to understand the flow generation mechanism in the simulation.

\section{Model}

In this section, brief description of the model for a nonlinear simulation is given. Then, the characteristics of obtained nonlinear saturation data are explained.

\subsection{Model equations}

A three-dimensional simulation of resistive drift wave turbulence in a linear device is performed using the NLD. The geometry of the plasma is a simple cylindrical shape, 
and the magnetic field has only the axial component with a uniform intensity. In linear device PANTA, detail measurements are carried out to clarify the nonlinear mechanisms in turbulent plasmas [15]. According to experiments in PANTA device, we focus on high density $n_{\mathrm{e}}>$ $1 \times 10^{19}\left[\mathrm{~m}^{-3}\right]$ with low temperature $T_{\mathrm{e}}<5[\mathrm{eV}]$ plasmas in an argon discharge, and take the effect of neutral particles into account. The code is based on the three-field (density, electrostatic potential, and parallel velocity of electrons) reduced fluid equations, which consists of the continuity equation, the charge conservation equation, and the momentum conservation equation [14]:

$$
\begin{gathered}
\frac{\partial N}{\partial t}=[N, \phi]-V \nabla_{\|} N-\nabla_{\|} V+\mu_{N} \nabla_{\perp}^{2} N+S_{N}, \\
\frac{\partial \nabla_{\perp}^{2} \phi}{\partial t}=\left[\nabla_{\perp}^{2} \phi, \phi\right]-V \nabla_{\|} N-v_{\mathrm{in}} \nabla N \cdot \nabla_{\perp} \phi- \\
\nabla N \cdot \frac{\mathrm{d} \nabla_{\perp} \phi}{\mathrm{d} t}-v_{\mathrm{in}} \nabla_{\perp}^{2} \phi-\nabla_{\|} V+\mu_{w} \nabla_{\perp}^{4} \phi, \\
\frac{\partial V}{\partial t}=[V, \phi]+\frac{M}{m_{\mathrm{e}}}\left(\nabla_{\|} \phi-\nabla_{\|} N\right)-v_{\mathrm{e}} V+\mu_{V} \nabla_{\perp}^{2} V,
\end{gathered}
$$

where $N=\ln \left(n / n_{0}\right), n$ is the density, $n_{0}$ is the density at $r=0, \phi$ is the normalized potential by electron temperature, and $V$ is the electron parallel velocity normalized by the sound velocity. The convective derivative is written by using the Poisson bracket $[\phi$,$] . The particle source term$ is represented by $S_{N}$, and $M / m_{\mathrm{e}}$ is mass ratio of the ion and electron, $v_{\text {in }}$ is the ion-neutral collision frequency, $v_{\mathrm{e}}$ is the sum of the ion-electron and electron-neutral collision frequency, and $\left\{\mu_{N}, \mu_{W}, \mu_{V}\right\}$ is a set of viscosities. It should be noted that the collision frequency $\nu_{\text {in }}$ and viscosity $\mu_{W}$ works as damping of $\mathrm{ZF}$ so that they are the controlling parameters of turbulent states [8]. The derivative $\nabla_{\|}$represents the derivative in the axial direction. The ion cyclotron frequency and Larmor radius measured by the electron temperature are used for normalization of the time and space, respectively. The equations are solved using spectral expansion with the periodic boundary condition in the azimuthal and axial direction. The boundary condition in the radial direction are set to $f=0$ at $r=0, a$ when $m \neq 0$, and $\partial f / \partial r=0$ at $r=0, f=0$ at $r=a$ when $m=0$, where $m$ is the azimuthal mode number, $f$ implies $\{N, \phi, V\}$, and $r=a$ gives an outer boundary of the plasma column.

The set of the model equations in this study is the extension of Hasegawa-Wakatani equations to include the effect of neutral particles and the dynamics of parallel electron velocity [14]. The Hasegawa-Wakatani equation can be deduced by the following replacement; $v_{\text {in }} \rightarrow 0, V=$ $\alpha \nabla_{\|}(\phi-N)$, where $\alpha$ is a constant related to resistivity. It should be noted that the electron parallel dynamics is important for the formation of the $(0,0)$ potential as described in the next section. Thus, we extended to include the electron parallel dynamics with its nonlinearity.

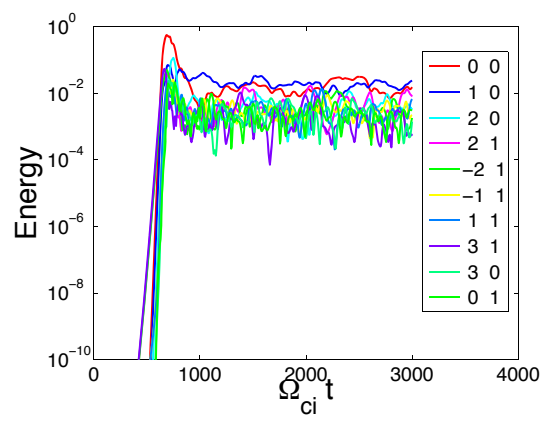

Fig. 1 Time evolutions of the energy of Fourier modes. The fluctuating electrostatic potential energies are shown.
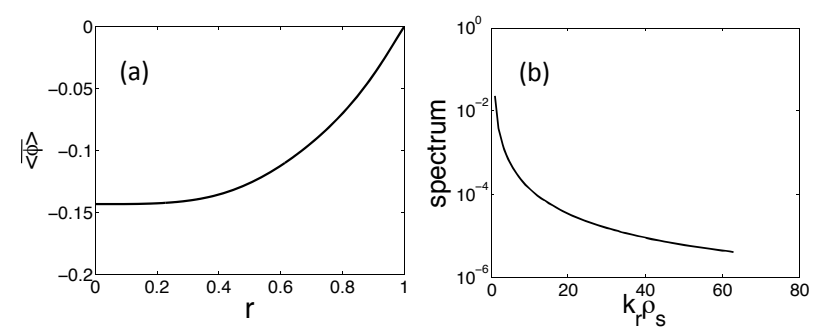

Fig. 2 (a) Radial profile of the mean potential $\phi_{00}$. (b) $k_{r}$ spectrum of the fluctuating component of $\phi_{00}$

\subsection{Nonlinear simulation}

A nonlinear simulation of resistive drift wave turbulence is performed by using parameters corresponding to PANTA experiments; $B=0.09[\mathrm{~T}], T_{\mathrm{e}}=3[\mathrm{eV}], a=$ $7[\mathrm{~cm}]$, length of the device $\lambda=4[\mathrm{~m}]$, and $\mu_{N}=1 \times 10^{-2}$, $\mu_{V}=\mu_{W}=1 \times 10^{-4}$. The collision frequency $v_{\text {in }}$ is a control parameter of instability here, which gives a selection rule of turbulent structure formations such as zonal flows and streamers [8]. The value is chosen as $v_{\text {in }}=0.005$, which gives zonal flow formation. The calculation is carried out with a fixed particle source, whose profile is given as

$$
S_{N}=\frac{4 S_{0} \mu_{N}}{L_{N}^{2}}\left\{1-\left(\frac{r}{L_{N}}\right)^{2}\right\} \exp \left[-\left(\frac{r}{L_{N}}\right)^{2}\right]
$$

where $S_{0}=5.0, L_{N}=6.7[\mathrm{~cm}]$. A density profile is peaked at $r=0$ by this particle source, and its gradient destabilizes the resistive drift wave. The simulation in this paper is performed with 256 grids in the radial directions, and Fourier modes $-8 \leq m \leq 8,-8 \leq n \leq 8$ are taken. The number of taken modes is not so large. That is because we need a long time series of data to carry out statistical analyses, and select nonlinear calculation with comparably short calculation time.

Nonlinear saturation is obtained as shown in Fig. 1. A variety of modes are excited with comparable energy, which gives a turbulent state. Components with $(m, n)=$ $(0,0)$ are also generated with the nonlinear couplings. The radial profile of mean $(0,0)$ potential and the $k_{r}$ spectrum of the fluctuating component are shown in Fig. 2, where 

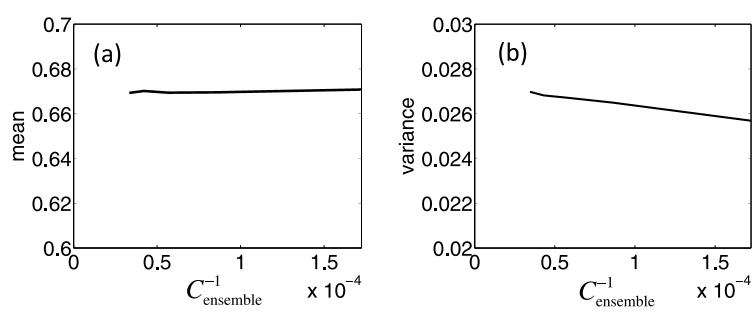

Fig. 3 Convergence of (a): the mean and (b): variance, against the inverse of ensemble number.

$\langle\ldots\rangle$ denotes the magnetic surface average. The timeaveraged potential has a gradient length comparable to the plasma radius, while the fluctuating component includes shorter spatial scale length. The mean sheared poloidal flow and fluctuating short wavelength flow, which are a macro-scale structure and zonal flow, respectively, co-exist in this turbulent state. The time evolution of the $(0,0)$ component of the potential shows that the radial wavenumber changes in time around $k_{r} \rho_{s}=0 \sim 1$ with the period of $\tau_{\mathrm{ZF}}=360 \Omega_{\mathrm{ci}}^{-1}$, which is 6 times longer than the typical drift wave period. It should be noted that a flute type mode with $(m, n)=(1,0)$ is driven in this simulation. This flute structure is linearly stable, but is driven by nonlinear couplings with drift waves and with centrifugal force by mean flow. The flute structure induces transport, and affects to flatten the density profile, which is different from the $\mathrm{ZF}$ and streamer. In this study, we investigate statistical properties of fluctuations of physical variables and their fluxes in the case of coexistence of $\mathrm{ZF}$ and flute structure.

\subsection{Convergence}

In order to carry out a statistical analysis of turbulent fluctuations, a long time evolution of turbulence for the duration of $62000 \Omega_{\mathrm{ci}}^{-1}$ (more than 1000 times of typical drift wave period) is calculated. Convergence of the mean and variance of each quantity is evaluated. Figure 3 shows the convergence of the flux-surface averaged density against ensemble number $C_{\text {ensemble }}$. The converged values can be estimated by linear fitting.

\section{Statistical Behavior of Fluxes}

In this section, dynamical balance for the formation of the $(0,0)$ components is analyzed. The mean value changes in time, which is affected by the dynamics of turbulent particle and momentum fluxes. The PDF is calculated to describe the dynamics.

\subsection{Formation of the $(0,0)$ components}

The roles of the fluxes on the formation of the $(m, n)$ $=(0,0)$ fields are described in this subsection. Time evolutions of the $(0,0)$ fields obey the flux-surface average of Eq. (1) and (2);
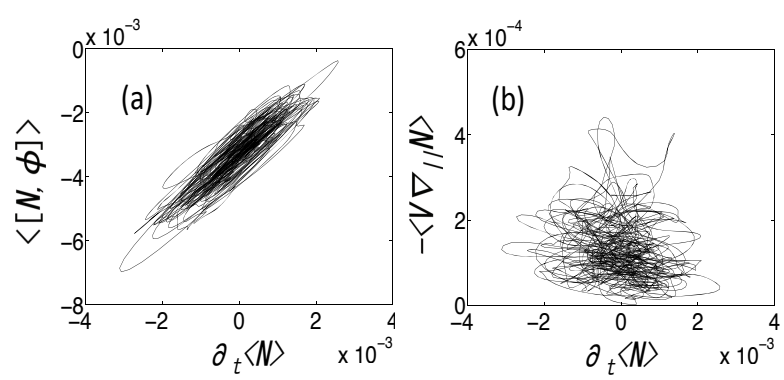

Fig. 4 Lissajous of time derivative of magnetic surface averaged density against (a) radial derivative of radial particle flux and (b) axial derivative of parallel particle flux.

$$
\begin{aligned}
\frac{\partial\langle N\rangle}{\partial t}=-\frac{1}{r} & \frac{\partial}{\partial r}\left(r\left\langle\Gamma_{r}\right\rangle\right)-\left\langle V \nabla_{\|} N\right\rangle+\mu_{N} \nabla_{\perp}^{2}\langle N\rangle+S, \\
\frac{\partial\left\langle\nabla_{\perp}^{2} \phi\right\rangle}{\partial t}= & \left\langle\left[\nabla_{\perp}^{2} \phi, \phi\right]\right\rangle-\left\langle V \nabla_{\|} N\right\rangle-v_{\text {in }}\left\langle\nabla N \cdot \nabla_{\perp} \phi\right\rangle \\
& -\left\langle\nabla N \cdot \frac{\mathrm{d} \nabla_{\perp} \phi}{\mathrm{d} t}\right\rangle-v_{\text {in }}\left\langle\nabla_{\perp}^{2} \phi\right\rangle+\mu_{w}\left\langle\nabla_{\perp}^{4} \phi\right\rangle,
\end{aligned}
$$

where, the particle flux is defined as

$$
\left\langle\Gamma_{r}\right\rangle=-\sum_{m n} \frac{m}{r} \operatorname{Im}\left[\phi_{m n} N_{m n}^{*}\right] .
$$

First, the roles of nonlinear terms on the density fluctuations are investigated. Nonlinear forces that drive fluctuations are the first and second terms in RHS of Eq. (5). In order to clarify their contributions, the relationships between the temporal change of the density and the nonlinear terms at $r / a=0.5$ are plotted in Fig. 4. Strong correlation with the radial derivative of the radial particle flux is confirmed, whereas that with the parallel nonlinearity is weak. This tendency is same in the other radial positions.

Next, the relationship of the vorticity is investigated. As in Eq. (6), there exist 4 nonlinear terms in the evolution equation of the vorticity. Although no momentum source is introduced, the vorticity has mean sheared component as in Fig. 2 (a). Figure 5 illustrates the radial profile of the time-averaged vorticity, and nonlinear contributions to its formation. The scale length of the vorticity is comparable to the plasma radius. The drift wave amplitude has a peak around $r / a \sim 0.8$, where the density profile is steep. Since the mean vorticity is generated by the nonlinear coupling of drift waves, the mean vorticity has a peak around $r / a \sim 0.8$. There exist finite driving forces with roughly constant except for the region near the edge. In this case, both the momentum flux and the parallel nonlinearity have comparable contributions. This result indicates the importance of the 3-dimensional structure of turbulent fields even in cylindrical plasmas [9].

For the evolution of the fluctuating components, the relationships between the temporal change of the vorticity and the nonlinear terms shows strong correlation only with the convective derivative term, as in Fig. 6. The convective 


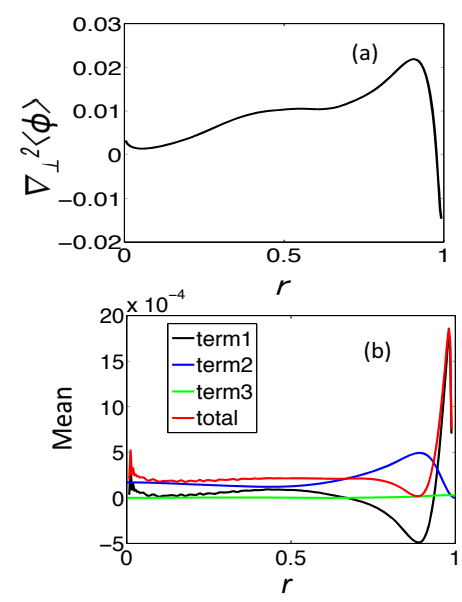

Fig. 5 Radial profiles of (a) the vorticity, and (b) nonlinear terms in the vorticity equation. Here, "term1-3" represent the first, second and third terms in Eq. (6), respectively, and "total" is the sum of these terms.
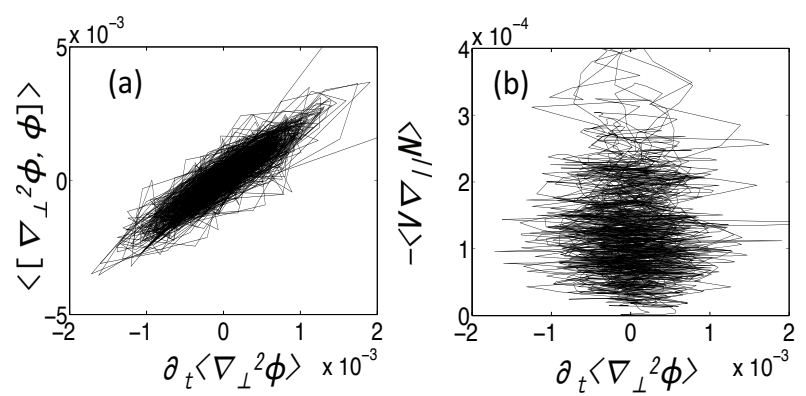

Fig. 6 Lissajous of time derivative of magnetic surface averaged vorticity against spatial derivatives of (a) the momentum flux, and (b) parallel particle flux term.

derivative term is related to the momentum flux,

$$
\langle\Pi\rangle=\sum_{m n} \frac{m}{r} \operatorname{Im}\left[\phi_{m n}^{*} \frac{\partial \phi_{m n}}{\partial r}\right],
$$

which is investigated in the next subsections.

\subsection{PDFs of particle and momentum fluxes}

As shown in the previous subsection, the particle and momentum fluxes have dominant contributions to variations of the fluctuations. In this subsection, the distribution of the fluxes in the temporal change is evaluated by use of PDFs. The PDFs of the fluxes at different several radial locations are shown in Fig. 7. The radial particle flux has a positive tail, which indicates positive spikes with large amplitudes occur more frequently than negative events. The momentum flux has a negative tail, but the deviation from the Gaussian is small. There is little difference in different radial locations. That is because the dominant contribution comes from the fluctuations with small radial wavelengths in this simulation.

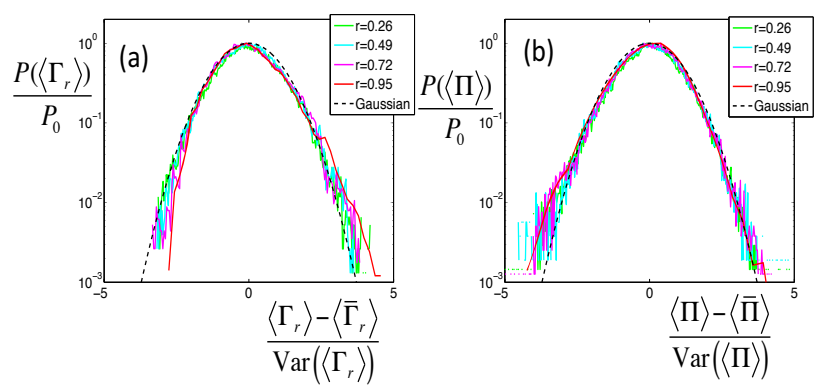

Fig. 7 PDFs of (a) the radial and (b) momentum flux at different radial locations. The origin and unit of the horizontal axis is set to be the mean value and variance of each, respectively.

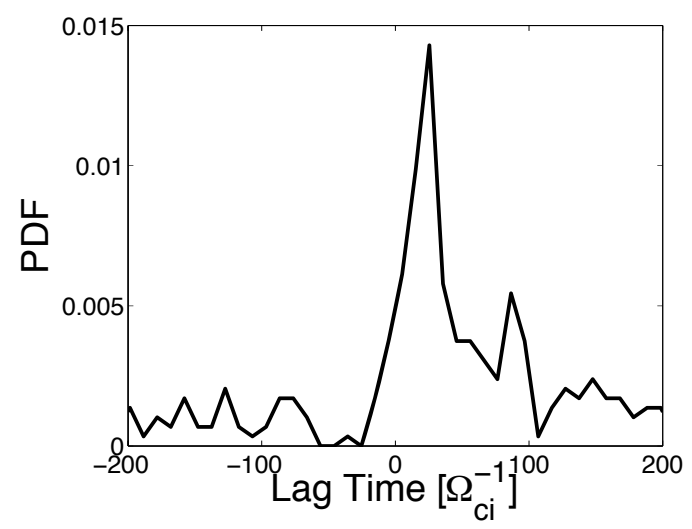

Fig. 8 PDF of time lags between $\left\langle\Gamma_{r}\right\rangle$ and $\langle\Pi\rangle$.

\subsection{Relation between particle and momen- tum fluxes}

Here, the causal relation between particle and momentum is discussed with the Joint-PDF. In order to clarify the causal relation between particle and momentum fluxes, the time lags of their variation are calculated by the crosscorrelation technique. Figure 8 shows a PDF of the time lags between $\left\langle\Gamma_{r}\right\rangle$ and $\langle\Pi\rangle$. The number of time windows with the time delay is counted from approximately 300 data windows. Maximum cross-correlation between $\left\langle\Gamma_{r}\right\rangle$ and $\langle\Pi\rangle$ is found to be around $0.2-0.4$. The significant peak can be seen at $\tau_{\text {lag }}=25 \Omega_{\mathrm{ci}}^{-1}$, which corresponds to 0.4 times of the typical drift wave period. The positive peak indicates that the change of $\left\langle\Gamma_{r}\right\rangle$ precedes that of $\langle\Pi\rangle$. It suggests that the density profile changes at first by the particle flux, and the changes affect the flow generation with the momentum flux.

A joint PDF between particle and momentum fluxes is constructed for the pair variables $P\left(\left\langle\Gamma_{r}(t)\right\rangle,\left\langle\Pi\left(t+\tau_{\text {lag }}\right)\right\rangle\right)$ with taking the time lag into consideration in Fig. 9. The contour of the joint PDF deviates from the circle shape, and clearly shows the correlation. The correlation exists in the wide range of their amplitudes from small events around the mean value to rare large events far from the mean. 


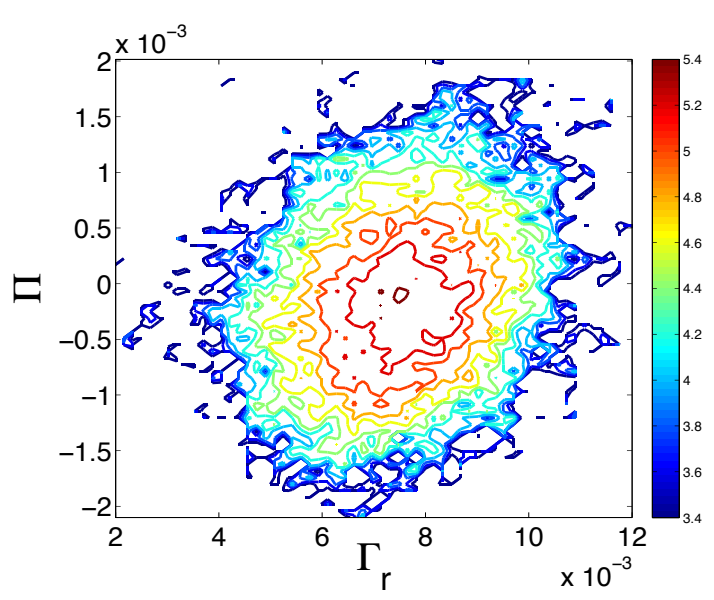

Fig. 9 Joint PDF between $\left\langle\Gamma_{r}\right\rangle$ and $\langle\Pi\rangle$.

\section{Summary}

The nonlinear simulation of resistive drift wave turbulence in a cylindrical plasma is performed by use of the NLD code. The nonlinear saturation state is accompanied by the formation of mean poloidal sheared flow and zonal flow. Long time evolution of the turbulence is obtained for 62000 times of ion cyclotron period, which corresponds to around 1000 times of the typical drift wave period. The convergence of values such as mean and variance is checked with obtained ensembles. The roles of the radial flux and parallel nonlinearity on the formation of the $(0,0)$ structure are studied by following the time evolution of each nonlinearities. The radial particle flux and momentum flux are important in the evolution of fluctuating components. The relationship between the radial particle flux and momentum flux is investigated by the cross correlation analysis. The particle flux is found to lead momen- tum flux for 0.4 times of the typical drift wave period with a large temporal variance, demonstrating that the density variation due to the radial particle flux is the primary drive for the fluctuations. This time delay is of the same order of magnitude compared to the experimental observation [6]. In this way, it is possible to investigate global nonlinear interactions with 3-dimensional time series data.

\section{Acknowledgement}

This work is partly supported by a grant-in-aid for scientific research of JSPS, Japan (24740372, 21224014, 22840034,23244113 ) and by the collaboration programs of NIFS (NIFS11KNST013, NIFS10KOAP023) and of the RIAM of Kyushu University and Asada Science foundation.

[1] A. Yoshizawa et al., Plasma and Fluid Turbulence (IoP, England, 2002).

[2] J.A. Krommes, Phys. Reports 360, 1-352 (2002).

[3] E.J. Kim and P.H. Diamond, Phys. Rev. Lett. 88, 225002 (2002).

[4] V. Naulin et al., Phys. Lett. A 321, 355 (2004).

[5] B.A. Carreras et al., Phys. Rev. Lett. 83, 3653 (1999).

[6] Y. Nagashima et al., Phys Plasmas 18, 070701 (2011).

[7] N. Kasuya et al., International Toki Conference, O5-3 (2012).

[8] N. Kasuya et al., Phys. Plasmas 15, 052302 (2008).

[9] A. Hasegawa et al., Phys. Rev. Lett. 50, 682 (1983).

[10] A. Hasegawa et al., Phys. Rev. Lett. 57, 1581 (1987).

[11] D. Biskamp et al., Phys. Rev. Lett. 74, 706 (1995).

[12] H. Sugama et al., Phys. Fluid 31, 1601 (1988).

[13] B. Friedman et al., Phys. Plasmas 19, 102307 (2012).

[14] N. Kasuya et al., J. Phys. Soc. Jpn. 76, 044501 (2007).

[15] S. Oldenbürger et al., Plasma Phys. Control. Fusion 54, 055002 (2012). 